\title{
Acúmulo de macronutrientes por plantas daninhas e de milho cultivadas em convivência em solo com diferentes manejos de fertilidade ${ }^{1}$
}

\section{Accumulation of macronutrients by weed and corn in coexistence in soil with different fertility managements}

\author{
Christiane Augusta Diniz Melo $^{2 *}$; Fernanda Aparecida Rodrigues Guimarães 3 ; \\ Valdinei Araújo Gonçalves 3 ; Sarah da Silva Benevenute \\ Giselle Lima Ferreira ${ }^{5}$, Lino Roberto Ferreira ${ }^{6}$; Francisco Affonso Ferreira ${ }^{6}$
}

\begin{abstract}
Resumo
Nos sistemas agrícolas o entendimento da interferência de plantas daninhas sobre as culturas é de grande importância. No entanto, pouco se sabe sobre a influência do manejo de fertilidade do solo nas relações de competição entre plantas. Assim, objetivou-se avaliar os efeitos da interferência mútua entre plantas daninhas e de milho no acúmulo de macronutrientes em solo com diferentes manejos de fertilidade. O ensaio foi realizado em casa de vegetação, incluindo quatro manejos de fertilidade do solo (com silicato de cálcio e magnésio e adubação; com calcário e adubação; sem correção de acidez, mas com adubação; sem correção de acidez e sem adubação) e onze cultivos (cinco arranjos de competição entre Zea mays e as plantas daninhas Urochloa brizantha, Ipomoea grandifolia, Conyza canadensis, Hyptis suaveolens e Bidens pilosa, acrescido das seis plantas em monocultivo). Sob interferência de $U$. brizantha, o milho apresentou reduções superiores a $50 \%$ no conteúdo de todos os macronutrientes. Adicionalmente, $U$. brizantha e B. pilosa em competição com o milho apresentaram elevado acúmulo de nutrientes nas quatro condições de solo. Silicato de cálcio e magnésio e calcário exerceram influência variável sobre o acúmulo de nutrientes pelas plantas daninhas em monocultivo ou sob interferência. $\mathrm{O}$ milho cultivado no solo corrigido com a fonte silicatada sofreu maior concorrência com as plantas daninhas em relação ao cultivado no solo corrigido com calcário. $U$. brizantha foi a espécie mais danosa ao milho. B. pilosa apresentou elevado potencial para ciclagem de macronutrientes nos quatro manejos de fertilidade do solo.
\end{abstract}

Palavras-chave: Interferência, nutrição mineral, calcário, silicato de cálcio e magnésio

1 Parte da Dissertação de Mestrado da primeira autora, apresentada ao Dept ${ }^{\circ}$ de Fitotecnia,Universidade Federal de Viçosa, UFV, Viçosa, MG.

2 Enga Agra , Discente de Doutorado em Fitotecnia, UFV, Viçosa, MG. Bolsista CNPq. E-mail: christiane.melo@ufv.br

3 Eng ${ }^{\text {os }}$ Agr ${ }^{\text {os }}$, Discentes de Doutorado em Fitotecnia, UFV, Viçosa, MG. Bolsistas CAPES. E-mail: fernanda.guimarães@ufv.br; valdinei.goncalves@ufv.br

4 Eng ${ }^{\mathrm{a}}$ Agra , UFV, Viçosa, MG. E-mail: sbenevenute@gmail.com

5 Enga Florestal, M.e em Fitotecnia, UFV, Viçosa, MG. E-mail: gisellelima87@yahoo.com.br

${ }^{6}$ Eng $^{\text {os }}$ Agr $^{\circ s}$, Profs. Dr., Dept ${ }^{\circ}$ de Fitotecnia, UFV, Viçosa, MG. Bolsistas de produtividade em pesquisa do CNPq. E-mail: 1roberto@ufv.br; faffonso@ufv.br

* Autor para correspondência 


\begin{abstract}
The understanding of weed interference on crops is very important in agricultural systems. However, there is little known about the influence of soil fertility management in competitive relations between plants. The objective of this study was to evaluate the effects of mutual interference between weeds and corn on the content of macronutrients under different soil fertility managements. The experimental test was conducted in a greenhouse, considering four soil fertility managements (with calcium and magnesium silicate and fertilization; with limestone and fertilization; without correction of acidity, but with fertilization; without correction of acidity and without fertilization) and eleven crops (five arrangements of competition between Zea mays and the weeds Urochloa brizantha, Ipomoea grandifolia, Conyza canadensis, Hyptis suaveolens and Bidens pilosa, plus the six species in monoculture). Corn had reductions exceeding $50 \%$ in the content of all macronutrients under interference of $U$. brizantha. Additionally, $U$. brizantha and B. pilosa in competition with corn has high ability to extract macronutrients in the four soil conditions. Calcium and magnesium silicate and limestone influenced variable on nutrient accumulation by weeds in monoculture or under interference. Corn grown in soil amended with silicate suffered greater competition with weeds compared to grown in soil amended with lime. $U$. brizantha was the specie most damaging to the crop. B. pilosa showed high potential for nutrient cycling in the four soil fertility management.
\end{abstract}

Key words: Interference, mineral nutrition, limestone, calcium and magnesium silicate

\section{Introdução}

Os solos brasileiros, em geral, apresentam elevada acidez, sendo essa característica um dos principais fatores limitantes à produção agrícola. Dessa forma, a correção é prática essencial para a garantia do sucesso da produtividade das culturas, tendo como benefício além da neutralização da acidez do solo, o fornecimento de cálcio e magnésio e a redução da toxidez por alumínio.

Os silicatos de cálcio e magnésio, principais constituintes das escórias de siderurgia, apresentam efeito corretivo da acidez do solo similar aos calcários. Possuem macro e micronutrientes e ainda silício, considerado como elemento benéfico para as plantas. Adicionalmente, algumas fontes de silicato são mais solúveis que o calcário, apresentando, portanto, maior potencial para a correção da acidez do solo em profundidade (RAMOS et al., 2006).

A acidez do solo pode afetar a produtividade do milho por restringir o crescimento radicular em decorrência de teores tóxicos de $\mathrm{Al}^{3+}$ e deficiência

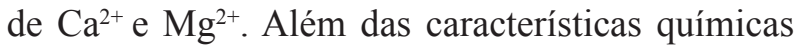
do solo, a convivência da cultura com plantas daninhas, principalmente nas fases iniciais de desenvolvimento, pode resultar em prejuízos à produção e operacionalização da colheita.
A cultura do milho, apesar de ser considerada competitiva por sombrear intensamente o solo, é afetada pela interferência de plantas daninhas, reduzindo o crescimento e a produtividade de grãos (CONSTANTIN et al., 2007). De modo geral, as plantas daninhas apresentam grande capacidade adaptativa e competitiva, além de aspectos positivos como proteção do solo e ciclagem de nutrientes (SILVA et al., 2007).

As plantas daninhas requerem, para seu desenvolvimento, os mesmos fatores de crescimento exigidos pela cultura do milho, estabelecendo um processo competitivo quando se desenvolvem conjuntamente. Neste contexto, a competição por nutrientes é de grande importância, pois esses, na maioria das vezes, são limitados. As perdas ocasionadas na cultura do milho em razão da interferência imposta pelas plantas daninhas têm sido descritas na ordem de $13,1 \%$, podendo chegar a $85 \%$ nos casos em que não tenha sido realizado nenhum método de controle (CARVALHO et al., 2007).

O entendimento da competição entre espécies de plantas é de fundamental importância nos sistemas agropecuários, notadamente onde são feitas associações entre plantas com diferentes 
características e habilidades competitivas. A competição entre plantas ocorre tanto abaixo como acima do solo e deve-se considerar que existe uma associação entre estes dois ambientes (ZANINE; SANTOS, 2004). Ademais, é importante conhecer, sob diversos manejos de fertilidade do solo, a capacidade de extração e acúmulo de nutrientes apresentado pelas plantas daninhas quando não estão em competição, de modo que o seu potencial de ciclagem e fornecimento de nutrientes possa ser considerado nos programas de manejo integrado.

A nutrição e a produtividade das culturas são afetadas pelas modificações químicas do solo causadas pela adição de corretivos de acidez e adubação. Estudos comparativos de fontes de corretivo de acidez são comuns na literatura, contudo, não há trabalhos sobre a possível influência da sua utilização sobre as relações de competição entre plantas daninhas e o milho. Diante disso, objetivouse avaliar os efeitos da interferência mútua entre plantas daninhas e de milho no conteúdo relativo de macronutrientes em solo com diferentes manejos de fertilidade.

\section{Material e Métodos}

\section{Histórico da área de coleta das amostras de solo}

A área de coleta das amostras de solo está situada no município de Cajuri, MG, localizada no terço médio de uma encosta com altitude de 678,74 m. Desde novembro de 2008, como forma de recuperação da pastagem degradada, a área é conduzida com sistemas de Integração Lavoura e Pecuária. No início das atividades em 2008 o solo, classificado como Latossolo Vermelho-Amarelo Distrófico, com textura argilosa, apresentava as seguintes características químicas, na profundidade de 0-20 cm: $\mathrm{pH}$ (água) $=4,4$; matéria orgânica= 2,10 dag kg-1; $\mathrm{P}=1,9 \mathrm{mg} \mathrm{dm}^{-3}, \mathrm{~K}=14,0 \mathrm{mg} \mathrm{dm}^{-3}$; $\mathrm{Ca}^{+2}, \mathrm{Mg}^{+2}, \mathrm{Al}^{+3}, \mathrm{H}+\mathrm{Al}, \mathrm{SB}$ e $\mathrm{CTC}_{\text {efetiva }}=0,2,0,1$, $1,8,8,75,0,34$ e $2,14 \mathrm{cmol}_{\mathrm{c}} \mathrm{dm}^{-3}$, respectivamente; $\mathrm{V}=4 \%$ e $\mathrm{m}=84 \%$.
Para implantação do sistema de consorciação foi realizada uma roçada da vegetação existente e distribuição a lanço, de calcário (calcário dolomítico, dose de 5,68 t ha ${ }^{-1}$ - PRNT 104,8\%) ou silicato de cálcio e magnésio (AgroSilício ${ }^{\circledR}$, dose de 7,01 t ha-1 - PRNT 85\%), conforme recomendação baseada em análise de solo, 60 dias antes da semeadura simultânea do milho (híbrido DKB 390) e da braquiária (Urochloa brizantha cv. Marandú). As necessidades de calcário e de silicato de cálcio e magnésio foram calculadas para neutralizar o $\mathrm{Al}^{3+}$, considerando a saturação crítica do $\mathrm{Al}^{3+}$ de $15 \%$, e elevar os teores de $\mathrm{Ca}^{2+}+\mathrm{Mg}^{2+}$ até $3 \mathrm{cmol}_{\mathrm{c}} \mathrm{dm}^{-}$

${ }^{3}$, de acordo com a exigência da cultura do milho (ALVES et al., 1999).

Em parcelas de $64 \mathrm{~m}^{2}(6,4 \times 10 \mathrm{~m})$ com oito fileiras de milho e braquiária (mesma linha e semeio simultâneo), espaçadas de $0,8 \mathrm{~m}$, foram implantados quatro tratamentos que constaram da aplicação isolada de duas fontes de corretivo do solo (calcário ou silicato de cálcio e magnésio), distribuídos em superfície, e da presença de adubação química, além de duas testemunhas (sem correção, mas com adubação e sem correção e sem adubação). $\mathrm{O}$ delineamento experimental utilizado foi em blocos ao acaso com três repetições.

As adubações de plantio e cobertura nos anos de 2008 foram realizadas com a utilização de $500 \mathrm{~kg}$ $\mathrm{ha}^{-1}$ da formulação NPK 8-24-12 + 0,4\% de Zn e $0,2 \%$ de $\mathrm{B}$ e $500 \mathrm{~kg} \mathrm{ha}^{-1}$ de 30-0-10, respectivamente. Após a colheita do milho a área foi pastoreada por bovinos até 20 dias antes do próximo cultivo. No segundo ano (2009) e no terceiro (2010) foram realizadas novamente a dessecação, a semeadura simultânea de milho e braquiária e adubação de plantio e cobertura seguindo a mesma metodologia do primeiro ano.

\section{Etapa experimental referente ao presente trabalho}

Amostras de solo foram coletadas naprofundidade de $0-10 \mathrm{~cm}$, em abril de 2011, nos tratamentos estabelecidos a campo em 2008. Posteriormente à 
coleta do solo, as amostras foram caracterizadas quimicamente (Tabela 1) e identificadas como: AA - recebeu correção de acidez com silicato de cálcio e magnésio e adubação; CA - recebeu correção de acidez com calcário e adubação; SA - sem correção de acidez, mas com adubação; SS - sem correção de acidez e sem adubação.

Tabela 1. Características químicas das amostras de solo coletadas na profundidade de 0-10 cm no município de Cajuri-MG, 2011.

\begin{tabular}{ccccccccccccc}
\hline $\begin{array}{l}\text { Manejos de } \\
\text { fertilidade }\end{array}$ & $\mathrm{pH}$ & $\mathrm{P}$ & $\mathrm{K}$ & $\mathrm{Ca}^{2+}$ & $\mathrm{Mg} \mathrm{dm}^{2+}$ & $\mathrm{Al}^{3+}$ & $\mathrm{H}+\mathrm{Al}$ & $\mathrm{SB}$ & $\mathrm{CTC}(\mathrm{t})$ & $\mathrm{V}$ & $\mathrm{m}$ & M.O. \\
\hline $\mathrm{AA}^{(1)}$ & 5,9 & 8,6 & 71 & 3,8 & 1,5 & 0,0 & 4,79 & 5,48 & 5,48 & 53 & 0 & 3,3 \\
$\mathrm{CA}$ & 5,9 & 10,3 & 74 & 3,8 & 1,1 & 0,0 & 5,12 & 5,09 & 5,09 & 50 & 0 & 2,8 \\
$\mathrm{SA}$ & 4,8 & 11,6 & 70 & 1,1 & 0,6 & 0,8 & 9,73 & 1,88 & 2,68 & 16 & 30 & 3,7 \\
$\mathrm{SS}$ & 4,5 & 2,2 & 36 & 0,3 & 0,2 & 1,9 & 11,72 & 0,59 & 2,49 & 5 & 76 & 3,5 \\
\hline
\end{tabular}

${ }^{(1)} \mathrm{AA}$ - com correção de acidez utilizando silicato de cálcio e magnésio e adubação; CA - com correção de acidez utilizando calcário e adubação; SA - sem correção de acidez, mas com adubação; SS - sem correção de acidez e sem adubação.

Fonte: Elaboração dos autores.

Foram avaliadas cinco espécies de plantas daninhas: Urochloa brizantha (braquiarão), Ipomoea grandifolia (corda-de-viola), Hyptis suaveolens (cheirosa), Conyza canadensis (buva) e Bidens pilosa (picão-preto) escolhidas pela comum ocorrência na área experimental onde foram realizadas as coletas de solo.

$\mathrm{O}$ experimento foi instalado e conduzido em casa de vegetação, em esquema fatorial 4 x 11, no delineamento em blocos casualizados com três repetições. Os fatores constituíram-se de quatro manejos de fertilidade de solo e onze cultivos, os quais corresponderam a cinco arranjos de competição entre o milho e as plantas daninhas, além das seis espécies em monocultivo.

Previamente ao preenchimento dos vasos, todas as amostras de solo foram adubadas com sulfato de amônio $\left(0,20 \mathrm{~g} \mathrm{dm}^{-3} \mathrm{de} \mathrm{N}\right)$, superfosfato simples $\left(0,20 \mathrm{~g} \mathrm{dm}^{-3} \mathrm{de}_{2} \mathrm{O}_{5}\right)$ e cloreto de potássio $\left(0,18 \mathrm{~g} \mathrm{dm}^{-3}\right.$ de $\left.\mathrm{K}_{2} \mathrm{O}\right)$, mantendo-se distintas as características provenientes da aplicação das fontes de correção de acidez de solo e adubação residual do campo.

Logo após, sementes de milho (híbrido 390 VT Pro) foram distribuídas simultaneamente às das plantas daninhas nos vasos contendo $5,5 \mathrm{~kg}$ de solo, restando em cada vaso, após desbaste, uma planta de milho no centro com mais quatro plantas daninhas da mesma espécie, formando os cinco arranjos de competição. A unidade experimental foi constituída por um vaso contendo uma planta de milho ou uma planta daninha isolada ou em competição. As plantas foram irrigadas conforme necessidade, de modo a manter a umidade do solo próxima à capacidade de campo.

Decorridos dez dias da semeadura, com a emergência de todas as espécies, procedeu-se ao desbaste, deixando-se o milho em convivência com as plantas daninhas por mais 55 dias. Nesta data, as plantas de milho apresentavam 6-8 folhas completamente expandidas e as plantas daninhas, com exceção de $B$. pilosa e $H$. suaveolens que se encontravam em estádio de florescimento, apresentavam-se em fase vegetativa.

Após o período de convivência, a parte aérea das plantas daninhas e de milho foi seccionada rente ao solo, acondicionada em sacos de papel e levado para estufa de circulação forçada de ar $\left(65^{\circ} \mathrm{C}\right)$, onde foram deixadas até atingir massa constante. Posteriormente, todo o material seco da parte aérea de milho e das plantas daninhas foi moído, em moinho analítico, homogeneizado e submetido 
à digestão nítricoperclórica para determinação das concentrações de fósforo $(\mathrm{P})$, pelo método da vitamina C modificado (BRAGA; DEFELIPO, 1974); de potássio (K) por fotometria de chama (SARRUGE; HAAG, 1974); de cálcio (Ca) e magnésio $(\mathrm{Mg})$ por espectrofotometria de absorção atômica (MALAVOLTA; VITTI; OLIVEIRA, 1997) e determinação de enxofre (S) em espectrofotômetro a $725 \mathrm{~nm}$ (MALAVOLTA; VITTI; OLIVEIRA, 1997). Após digestão sulfúrica foi determinado o teor de nitrogênio total $(\mathrm{N})$ pelo método Kjeldahl (YASUHARA; NOKIHARA, 2001).

A partir da massa da matéria seca da parte aérea $(\mathrm{kg})$ e de seus respectivos teores de nutrientes (dag $\mathrm{kg}^{-1}$ ), procedeu-se o cálculo do conteúdo de macronutriente na parte aérea dessas plantas (mg) cultivadas nas quatro condições de solo.

Para interpretação dos resultados, os dados obtidos foram submetidos à análise de variância pelo teste $\mathrm{F}(\mathrm{p} \leq 0,05)$. Além do desdobramento da interação significativa, optou-se por apresentar também o estudo das comparações de cada fator isolado, sendo as médias comparadas pelo teste de Duncan $(\mathrm{p} \leq 0,05)$.

\section{Resultados e Discussão}

O conteúdo de macronutrientes na parte aérea de plantas daninhas e de milho foi influenciado pela interação entre os manejos de fertilidade do solo e as espécies submetidas ou não à interferência. Os teores de nutrientes encontrados foram convertidos em conteúdo na parte aérea das plantas, já que, determinada planta daninha pode apresentar os maiores teores de nutrientes, mas, devido à sua baixa produção de matéria seca, pode apresentar os menores conteúdos destes (RONCHI et al., 2003).

O potencial de extração e utilização de nutrientes de cada planta daninha e do milho livre de interferência no solo submetido a diferentes manejos de fertilidade pode ser verificado na Tabela 2. Segundo Karam, Melhorança e Oliveira (2006) o milho, mesmo sendo eficiente na absorção, não consegue acumular nutrientes como as plantas infestantes fazem em seus componentes vegetativos. No entanto, constatou-se que, independente da condição de solo, em média, as espécies $B$. pilosa e Z. mays destacaram-se em relação às demais plantas daninhas, apresentando os maiores conteúdos na parte aérea de todos os macronutrientes (Tabela 2).

Como efeito dos manejos de fertilidade do solo, U. brizantha e B. pilosa obtiveram maiores acúmulos de $\mathrm{N}$ na parte aérea, sem correção de acidez e sem adubação no solo, o que evidencia que mesmo em condições de escassez de nutrientes e elevada saturação por alumínio, essas plantas apresentam flexibilidade adaptativa e elevada habilidade em extrair esse nutriente (Tabela 2), podendo representar para o milho e para outras espécies não-cultivadas, nessas condições de solo, bons competidores, principalmente por ocorrerem em altas densidades. Ainda neste contexto, apesar de poucos relatos na literatura, ressalta-se o grande potencial das plantas daninhas para a ciclagem de nutrientes, e considerando, em especial, a grande utilização de $U$. brizantha nos processos de renovação e recuperação de pastagens degradadas, em áreas com características químicas do solo desfavoráveis, tal fato pode conferir à forrageira menor exigência e dependência da aplicação de fertilizantes. 
Melo, C. A. D. et al.

Tabela 2. Conteúdo de macronutrientes na parte aérea de plantas de milho, Urochloa brizantha, Ipomoea grandifolia, Hyptis suaveolens, Conyza canadensis e Bidens pilosa, cultivadas livre de interferência, em solo com diferentes manejos de fertilidade.

\begin{tabular}{|c|c|c|c|c|c|}
\hline \multirow{2}{*}{ Espécies } & \multicolumn{4}{|c|}{$\mathbf{N}\left(\mathrm{mg}\right.$ planta $\left.^{-1}\right)$} & \multirow{2}{*}{ Média } \\
\hline & $\mathbf{A} \mathbf{A}^{(1)}$ & $\mathbf{C A}$ & $\mathbf{S A}$ & SS & \\
\hline Zea mays & $183,50 \mathrm{Bb}^{(2)}$ & $229,59 \mathrm{Aa}$ & $74,15 \mathrm{Db}$ & $113,88 \mathrm{Cc}$ & $150,28 \mathrm{~b}$ \\
\hline Urochloa brizantha & $144,39 \mathrm{Bc}$ & $119,25 \mathrm{Bb}$ & $34,72 \mathrm{Cc}$ & $204,75 \mathrm{Ab}$ & $125,78 \mathrm{c}$ \\
\hline Ipomoea grandifolia & $44,61 \mathrm{Bd}$ & $104,81 \mathrm{Abc}$ & $35,62 \mathrm{Bc}$ & $17,63 \mathrm{Bd}$ & $50,67 \mathrm{~d}$ \\
\hline Hyptis suaveolens & $54,30 \mathrm{Ad}$ & $28,81 \mathrm{Ad}$ & $26,66 \mathrm{Ac}$ & $31,00 \mathrm{Ad}$ & $35,19 \mathrm{~d}$ \\
\hline Conyza canadensis & 41,98 Ad & $36,42 \mathrm{Ad}$ & 41,79 Abc & $17,29 \mathrm{Ad}$ & $34,37 \mathrm{~d}$ \\
\hline Bidens pilosa & $228,81 \mathrm{Ba}$ & $78,18 \mathrm{Dc}$ & $146,80 \mathrm{Ca}$ & $274,32 \mathrm{Aa}$ & $182,03 \mathrm{a}$ \\
\hline Média & $116,27 \mathrm{~A}$ & $99,51 \mathrm{~B}$ & $59,96 \mathrm{C}$ & $109,81 \mathrm{AB}$ & \\
\hline CV (\%) & & & 22,05 & & \\
\hline \multirow{2}{*}{ Espécies } & \multicolumn{4}{|c|}{$\mathbf{P}\left(\mathrm{mg} \mathrm{planta}^{-1}\right)$} & \multirow{2}{*}{ Média } \\
\hline & $\mathbf{A A}$ & CA & SA & SS & \\
\hline Zea mays & $17,88 \mathrm{Ba}$ & $33,74 \mathrm{Aa}$ & $11,86 \mathrm{Cb}$ & $5,73 \mathrm{Dc}$ & $17,30 \mathrm{~b}$ \\
\hline Urochloa brizantha & $13,14 \mathrm{Ab}$ & $13,01 \mathrm{Ab}$ & $4,74 \mathrm{Cc}$ & $9,85 \mathrm{Bb}$ & $10,19 \mathrm{c}$ \\
\hline Ipomoea grandifolia & $2,58 \mathrm{BCd}$ & $9,72 \mathrm{Ac}$ & $3,33 \mathrm{Bd}$ & $0,66 \mathrm{Cd}$ & $4,07 \mathrm{e}$ \\
\hline Hyptis suaveolens & 4,50 Abcd & 4,87 Abd & $5,85 \mathrm{Ac}$ & $3,00 \mathrm{Bd}$ & $4,55 \mathrm{e}$ \\
\hline Conyza canadensis & $6,05 \mathrm{Bc}$ & $5,74 \mathrm{Bd}$ & $10,70 \mathrm{Ab}$ & $2,21 \mathrm{Cd}$ & $6,18 \mathrm{~d}$ \\
\hline Bidens pilosa & $18,25 \mathrm{Ba}$ & $11,65 \mathrm{Cbc}$ & $29,49 \mathrm{Aa}$ & $16,04 \mathrm{Ba}$ & $18,86 \mathrm{a}$ \\
\hline Média & $10,40 \mathrm{~B}$ & $13,12 \mathrm{~A}$ & $11,00 \mathrm{~B}$ & $6,00 \mathrm{C}$ & \\
\hline CV (\%) & & & 13,83 & & \multirow{3}{*}{ Média } \\
\hline \multirow{2}{*}{ Espécies } & \multicolumn{4}{|c|}{ K (mg planta $\left.{ }^{-1}\right)$} & \\
\hline & $\mathbf{A A}$ & $\mathbf{C A}$ & SA & SS & \\
\hline Zea mays & $268,71 \mathrm{Ba}$ & $497,34 \mathrm{Aa}$ & $159,81 \mathrm{Cb}$ & $111,08 \mathrm{Db}$ & $259,23 \mathrm{a}$ \\
\hline Urochloa brizantha & $134,31 \mathrm{Ac}$ & $101,78 \mathrm{Bb}$ & $48,83 \mathrm{Cc}$ & $103,53 \mathrm{Bb}$ & $97,11 \mathrm{c}$ \\
\hline Ipomoea grandifolia & $18,83 \mathrm{Be}$ & $63,30 \mathrm{Ac}$ & $26,69 \mathrm{Bc}$ & $8,90 \mathrm{Bc}$ & $29,43 \mathrm{de}$ \\
\hline Hyptis suaveolens & 34,25 Ade & $22,05 \mathrm{Ad}$ & $22,44 \mathrm{Ac}$ & $24,30 \mathrm{Ac}$ & $25,76 \mathrm{e}$ \\
\hline Conyza canadensis & $52,81 \mathrm{Ad}$ & $27,01 \mathrm{Ad}$ & $54,02 \mathrm{Ac}$ & $34,18 \mathrm{Ac}$ & $42,01 \mathrm{~d}$ \\
\hline Bidens pilosa & $205,08 \mathrm{Bb}$ & $121,50 \mathrm{Cb}$ & $263,00 \mathrm{Aa}$ & $201,18 \mathrm{Ba}$ & $197,77 \mathrm{~b}$ \\
\hline Média & $119,00 \mathrm{~B}$ & $138,83 \mathrm{~A}$ & $95,85 \mathrm{C}$ & $80,53 \mathrm{D}$ & \\
\hline CV (\%) & & & 16,50 & & \\
\hline \multirow{2}{*}{ Espécies } & \multicolumn{4}{|c|}{ Ca (mg planta $\left.{ }^{-1}\right)$} & \multirow{2}{*}{ Média } \\
\hline & $\mathbf{A A}$ & CA & SA & SS & \\
\hline Zea mays & $72,58 \mathrm{Bb}$ & $114,49 \mathrm{Aa}$ & $29,98 \mathrm{Cbc}$ & $16,47 \mathrm{Db}$ & $58,38 \mathrm{~b}$ \\
\hline Urochloa brizantha & $25,78 \mathrm{Ac}$ & $24,90 \mathrm{Ae}$ & $11,73 \mathrm{Bd}$ & $14,44 \mathrm{Bbc}$ & $19,21 \mathrm{~d}$ \\
\hline Ipomoea grandifolia & $17,69 \mathrm{Bc}$ & $65,69 \mathrm{Ac}$ & $22,86 \mathrm{Bc}$ & $5,14 \mathrm{Cc}$ & $27,84 \mathrm{c}$ \\
\hline Hyptis suaveolens & $23,63 \mathrm{Bc}$ & $35,71 \mathrm{Ad}$ & $36,55 \mathrm{Ab}$ & $9,51 \mathrm{Cbc}$ & $26,35 \mathrm{c}$ \\
\hline Conyza canadensis & $23,63 \mathrm{Bc}$ & $19,92 \mathrm{Be}$ & $33,74 \mathrm{Ab}$ & $8,26 \mathrm{Cbc}$ & $21,22 \mathrm{~d}$ \\
\hline Bidens pilosa & $104,10 \mathrm{Ba}$ & $96,61 \mathrm{Bb}$ & $140,74 \mathrm{Aa}$ & $74,82 \mathrm{Ca}$ & $104,07 \mathrm{a}$ \\
\hline Média & $44,45 \mathrm{~B}$ & $59,55 \mathrm{~A}$ & $45,93 \mathrm{~B}$ & $21,44 \mathrm{C}$ & \\
\hline CV (\%) & & & 14,10 & & \\
\hline
\end{tabular}


continuação

\begin{tabular}{|c|c|c|c|c|c|}
\hline \multirow{2}{*}{ Espécies } & \multicolumn{4}{|c|}{ Mg (mg planta $\left.{ }^{-1}\right)$} & \multirow{2}{*}{ Média } \\
\hline & $\mathbf{A A}$ & $\mathbf{C A}$ & SA & SS & \\
\hline Zea mays & $34,50 \mathrm{Ba}$ & $75,93 \mathrm{Aa}$ & $16,41 \mathrm{Cb}$ & $6,52 \mathrm{Db}$ & $33,34 \mathrm{a}$ \\
\hline Urochloa brizantha & $19,64 \mathrm{Ab}$ & $21,52 \mathrm{Ab}$ & $6,15 \mathrm{Bc}$ & $9,89 \mathrm{Bb}$ & $14,30 \mathrm{~b}$ \\
\hline Ipomoea grandifolia & 3,77 Ac & 9,56 Ac & $3,89 \mathrm{Ac}$ & $0,98 \mathrm{Ab}$ & $4,55 \mathrm{c}$ \\
\hline Hyptis suaveolens & $4,48 \mathrm{Ac}$ & $5,82 \mathrm{Ac}$ & $7,72 \mathrm{Ab}$ & $1,74 \mathrm{Ab}$ & $4,94 \mathrm{c}$ \\
\hline Conyza canadensis & 4,51 Ac & 3,07 Ac & $6,58 \mathrm{Ac}$ & $1,98 \mathrm{Ab}$ & $4,03 \mathrm{c}$ \\
\hline Bidens pilosa & 39,62 Aa & $19,68 \mathrm{Bb}$ & $43,41 \mathrm{Aa}$ & $25,80 \mathrm{Ba}$ & $32,13 \mathrm{a}$ \\
\hline Média & $17,75 \mathrm{~B}$ & $22,60 \mathrm{~A}$ & $14,03 \mathrm{~B}$ & $7,82 \mathrm{C}$ & \\
\hline CV (\%) & & & 36,40 & & \multirow{3}{*}{ Média } \\
\hline \multirow{2}{*}{ Espécies } & \multicolumn{4}{|c|}{ S (mg planta $\left.{ }^{-1}\right)$} & \\
\hline & $\mathbf{A A}$ & $\mathbf{C A}$ & SA & SS & \\
\hline Zea mays & $25,14 \mathrm{Aa}$ & $25,69 \mathrm{Aa}$ & $13,98 \mathrm{Ba}$ & $13,54 \mathrm{Ba}$ & $19,59 \mathrm{a}$ \\
\hline Urochloa brizantha & $12,45 \mathrm{Ac}$ & $10,08 \mathrm{Ac}$ & $3,12 \mathrm{Bb}$ & $8,46 \mathrm{Ab}$ & $8,53 \mathrm{c}$ \\
\hline Ipomoea grandifolia & $5,16 \mathrm{BCd}$ & $17,22 \mathrm{Ab}$ & $6,22 \mathrm{Bb}$ & $1,63 \mathrm{Cc}$ & $7,56 \mathrm{c}$ \\
\hline Hyptis suaveolens & 4,41 Ad & 4,42 Ade & $3,73 \mathrm{Ab}$ & $3,39 \mathrm{Ac}$ & $3,99 \mathrm{~d}$ \\
\hline Conyza canadensis & 5,31 Ad & $3,71 \mathrm{Ae}$ & $5,15 \mathrm{Ab}$ & $1,28 \mathrm{Ac}$ & $3,86 \mathrm{~d}$ \\
\hline Bidens pilosa & $17,27 \mathrm{Ab}$ & $8,14 \mathrm{Bcd}$ & $16,17 \mathrm{Aa}$ & $14,93 \mathrm{Aa}$ & $14,13 \mathrm{~b}$ \\
\hline Média & $11,62 \mathrm{~A}$ & $11,54 \mathrm{~A}$ & $8,06 \mathrm{~B}$ & $7,21 \mathrm{~B}$ & \\
\hline CV (\%) & & & 25,61 & & \\
\hline
\end{tabular}

${ }^{(1)} \mathrm{AA}$ - com correção de acidez utilizando silicato de cálcio e magnésio e adubação; CA - com correção de acidez utilizando calcário e adubação; SA - sem correção de acidez, mas com adubação; SS - sem correção de acidez e sem adubação. ${ }^{(2)}$ Médias seguidas pela mesma letra maiúscula na linha e minúscula na coluna não diferem entre si pelo teste de Duncan $(\mathrm{p}>0,05)$.

Fonte: Elaboração dos autores.

O conteúdo de $\mathrm{N}, \mathrm{P}, \mathrm{K}, \mathrm{Ca}, \mathrm{Mg}$ e $\mathrm{S}$ na parte aérea da maioria das plantas daninhas, em que houve efeito significativo do manejo de fertilidade do solo, foi substancialmente menor no solo sem correção de acidez e com adubação, em comparação aos demais, com exceção para B. pilosa que acumulou, quando cultivada nesta condição de solo, os maiores conteúdos de $\mathrm{P}, \mathrm{K}, \mathrm{Ca}, \mathrm{Mg}$ e $\mathrm{S}$, juntamente com $H$. suaveolens e $C$. canadensis que apresentaram destaque no conteúdo de $\mathrm{Ca}$ e $\mathrm{P}$ (Tabela 2). Zea mays foi afetada negativamente pela ausência de correção de acidez no solo, com baixo acúmulo de macronutrientes (Tabela 2).

Z. mays apresentou maior eficiência que as plantas daninhas no acúmulo dos macronutrientes no solo que recebeu calcário e adubação, destacandose também no solo que foi aplicado o silicato de cálcio e magnésio e adubação, exceto para $B$. pilosa em AA, com maior conteúdo na parte aérea de $\mathrm{P}$, $\mathrm{K}, \mathrm{Mg}$ e S (Tabela 2). Estes resultados encontram respaldo nas observações de Cury et al. (2012) que constatou, por meio da média dos três cultivares de milho avaliados, que a cultura do milho apresentou acúmulo de nutrientes semelhante ou superior aos obtidos pelas diferentes espécies de plantas daninhas. Em contrapartida, B. pilosa nos solos sem adição de fonte de correção de acidez (SA e SS) obteve maior conteúdo na parte aérea de $\mathrm{N}, \mathrm{P}, \mathrm{K}$, $\mathrm{Ca}$ e $\mathrm{Mg}$ que as plantas de milho e demais plantas daninhas (Tabela 2). Destaca-se a grande influência exercida pelas características químicas de solo, e a contribuição que algumas plantas podem conferir com a ciclagem de nutrientes e potencial redução do aporte de insumos externos, se consideradas nos programas de manejo integrado de plantas daninhas.

Verificaram-se reduções médias dos macronutrientes na parte aérea de plantas de milho sob interferência das plantas daninhas (Tabela 3). $U$. brizantha foi a planta que, independente dos manejos de fertilidade do solo, provocou maiores reduções 
no conteúdo de $\mathrm{N}, \mathrm{P}, \mathrm{K}, \mathrm{Ca}$ e $\mathrm{S}$ na parte aérea do milho, da ordem de 53,59,56, 59 e 55\%, respectiva e comparativamente à média da testemunha livre de interferência (Tabela 3). De modo geral, nas condições de solo com maior escassez nutricional menores extrações e acúmulos de macronutrientes pelas plantas de milho foram observados (Tabela 3 ).

Tabela 3. Conteúdo de macronutrientes na parte aérea de plantas de milho cultivadas sob interferência de Urochloa brizantha, Ipomoea grandifolia, Hyptis suaveolens, Conyza canadensis e Bidens pilosa em solo com diferentes manejos de fertilidade.

\begin{tabular}{|c|c|c|c|c|c|}
\hline \multirow{2}{*}{ Espécies } & \multicolumn{4}{|c|}{$\mathbf{N}($ mg planta-1) } & \multirow{2}{*}{ Média } \\
\hline & $\mathbf{A A}^{(1)}$ & CA & SA & SS & \\
\hline Zea mays & $183,50 \mathrm{Ba}^{(2)}$ & $229,59 \mathrm{Aa}$ & 74,15 Dabc & $113,88 \mathrm{Ca}$ & $150,28 \mathrm{a}$ \\
\hline Z. mays + Urochloa brizantha & $59,29 \mathrm{Ac}$ & $94,73 \mathrm{Ab}$ & $81,82 \mathrm{ABab}$ & $49,65 \mathrm{Bb}$ & $71,37 \mathrm{c}$ \\
\hline Z. mays +Ipomoea grandifolia & $92,61 \mathrm{Ac}$ & $75,91 \mathrm{Ab}$ & $95,10 \mathrm{Aa}$ & $114,51 \mathrm{Aa}$ & $94,53 \mathrm{~b}$ \\
\hline Z. mays +Hyptis suaveolens & $177,76 \mathrm{Aab}$ & $83,26 \mathrm{Bb}$ & $49,51 \mathrm{BCbc}$ & $44,36 \mathrm{Cb}$ & $88,75 \mathrm{bc}$ \\
\hline Z. mays + Conyza canadensis & $139,64 \mathrm{Ab}$ & $77,62 \mathrm{Bb}$ & $35,36 \mathrm{Cc}$ & $96,07 \mathrm{Ca}$ & $87,17 \mathrm{bc}$ \\
\hline Z. mays + Bidens pilosa & $142,23 \mathrm{Ab}$ & $113,32 \mathrm{Ab}$ & $53,16 \mathrm{Bbc}$ & $29,57 \mathrm{Bb}$ & $84,57 \mathrm{bc}$ \\
\hline Média & $132,51 \mathrm{~A}$ & $112,41 \mathrm{~B}$ & $64,87 \mathrm{C}$ & $74,67 \mathrm{C}$ & \\
\hline CV (\%) & \multicolumn{5}{|c|}{23,22} \\
\hline \multirow{2}{*}{ Espécies } & \multicolumn{4}{|c|}{$\mathbf{P}\left(\right.$ mg planta $\left.^{-1}\right)$} & \multirow{2}{*}{ Média } \\
\hline & $\mathbf{A A}$ & CA & SA & SS & \\
\hline Zea mays & $17,88 \mathrm{Ba}$ & $33,74 \mathrm{Aa}$ & $11,86 \mathrm{Ca}$ & $5,73 \mathrm{Dbc}$ & $17,30 \mathrm{a}$ \\
\hline Z. mays + Urochloa brizantha & $4,51 \mathrm{BCd}$ & $13,38 \mathrm{Ab}$ & $5,93 \mathrm{Bb}$ & $2,75 \mathrm{Cd}$ & $6,64 \mathrm{~d}$ \\
\hline Z. mays +Ipomoea grandifolia & $6,93 \mathrm{Bc}$ & 9,83 Ade & $11,15 \mathrm{Aa}$ & $5,19 \mathrm{Bc}$ & $8,27 \mathrm{c}$ \\
\hline Z. mays + Hyptis suaveolens & $8,49 \mathrm{Bbc}$ & 11,12 Acd & $7,11 \mathrm{BCb}$ & $5,72 \mathrm{Cbc}$ & $8,11 \mathrm{c}$ \\
\hline Z. mays + Conyza canadensis & $9,29 \mathrm{Bb}$ & $8,86 \mathrm{Be}$ & $5,38 \mathrm{Cb}$ & $15,23 \mathrm{Aa}$ & $9,79 \mathrm{~b}$ \\
\hline Z. mays + Bidens pilosa & $7,77 \mathrm{Bbc}$ & $13,06 \mathrm{Ab}$ & $6,83 \mathrm{Bb}$ & $7,45 \mathrm{Bb}$ & $8,78 \mathrm{bc}$ \\
\hline Média & $9,14 \mathrm{~B}$ & $15,00 \mathrm{~A}$ & $8,04 \mathrm{C}$ & $7,08 \mathrm{D}$ & \\
\hline CV (\%) & & & 12,79 & & \\
\hline \multirow{2}{*}{ Espécies } & \multicolumn{4}{|c|}{ K (mg planta $\left.{ }^{-1}\right)$} & \multirow{2}{*}{ Média } \\
\hline & AA & $\mathbf{C A}$ & SA & SS & \\
\hline Zea mays & $268,71 \mathrm{Ba}$ & $497,34 \mathrm{Aa}$ & $159,81 \mathrm{Cab}$ & $111,08 \mathrm{Da}$ & $259,23 \mathrm{a}$ \\
\hline Z. mays + Urochloa brizantha & $93,82 \mathrm{BCc}$ & $176,80 \mathrm{Ad}$ & $122,43 \mathrm{Bb}$ & $61,89 \mathrm{Cbcd}$ & $113,73 \mathrm{c}$ \\
\hline Z. mays + Ipomoea grandifolia & $104,04 \mathrm{Bc}$ & $174,24 \mathrm{Ad}$ & $167,08 \mathrm{Aa}$ & $82,26 \mathrm{Babc}$ & $131,90 \mathrm{bc}$ \\
\hline Z. mays + Hyptis suaveolens & $156,76 \mathrm{ABb}$ & $180,44 \mathrm{Ad}$ & $130,64 \mathrm{Bab}$ & $58,31 \mathrm{Ccd}$ & $131,54 \mathrm{bc}$ \\
\hline Z. mays + Conyza canadensis & $170,44 \mathrm{Bb}$ & $221,80 \mathrm{Ac}$ & $72,32 \mathrm{Cc}$ & $98,37 \mathrm{Cab}$ & $140,73 \mathrm{~b}$ \\
\hline Z. mays + Bidens pilosa & $141,08 \mathrm{Bb}$ & $266,79 \mathrm{Ab}$ & $85,44 \mathrm{Cc}$ & $36,79 \mathrm{Dd}$ & $132,53 \mathrm{~b}$ \\
\hline Média & $155,81 \mathrm{~B}$ & $252,90 \mathrm{~A}$ & $122,95 \mathrm{C}$ & $74,78 \mathrm{D}$ & \\
\hline CV (\%) & & & 14,28 & & \\
\hline \multirow{2}{*}{ Espécies } & \multicolumn{4}{|c|}{ Ca (mg planta $\left.{ }^{-1}\right)$} & \multirow{2}{*}{ Média } \\
\hline & $\mathbf{A A}$ & $\mathbf{C A}$ & SA & SS & \\
\hline Zea mays & $72,58 \mathrm{Ba}$ & $114,49 \mathrm{Aa}$ & $29,98 \mathrm{Cab}$ & $16,47 \mathrm{Dc}$ & $58,38 \mathrm{a}$ \\
\hline Z. mays + Urochloa brizantha & $20,48 \mathrm{Bc}$ & $47,49 \mathrm{Ab}$ & $18,57 \mathrm{Bcd}$ & $8,38 \mathrm{Cd}$ & $23,73 \mathrm{e}$ \\
\hline Z. mays +Ipomoea grandifolia & $24,49 \mathrm{Bbc}$ & $38,94 \mathrm{Ac}$ & $36,03 \mathrm{Aa}$ & $19,24 \mathrm{Bc}$ & $29,67 \mathrm{~cd}$ \\
\hline Z. mays + Hyptis suaveolens & $13,11 \mathrm{Cd}$ & $37,68 \mathrm{Ac}$ & $21,35 \mathrm{Bcd}$ & $34,10 \mathrm{Ab}$ & $26,56 \mathrm{de}$ \\
\hline Z. mays + Conyza canadensis & $30,58 \mathrm{Bb}$ & $33,45 \mathrm{Bc}$ & $14,75 \mathrm{Cd}$ & $118,99 \mathrm{Aa}$ & $49,44 \mathrm{~b}$ \\
\hline Z. mays + Bidens pilosa & $23,68 \mathrm{Cbc}$ & $51,61 \mathrm{Ab}$ & $24,82 \mathrm{Cbc}$ & $32,52 \mathrm{Bb}$ & $33,16 \mathrm{c}$ \\
\hline Média & $30,82 \mathrm{C}$ & $53,94 \mathrm{~A}$ & $24,25 \mathrm{D}$ & $38,28 \mathrm{~B}$ & \\
\hline CV (\%) & & & 12,06 & & \\
\hline
\end{tabular}


continuação

\begin{tabular}{|c|c|c|c|c|c|}
\hline \multirow{2}{*}{ Espécies } & \multicolumn{4}{|c|}{ Mg (mg planta $\left.{ }^{-1}\right)$} & \multirow{2}{*}{ Média } \\
\hline & $\mathbf{A A}$ & CA & SA & SS & \\
\hline Zea mays & $34,50 \mathrm{Ba}$ & $75,92 \mathrm{Aa}$ & $16,41 \mathrm{Cab}$ & $6,52 \mathrm{Cb}$ & $33,34 \mathrm{a}$ \\
\hline Z. mays + Urochloa brizantha & $11,14 \mathrm{Bb}$ & $23,97 \mathrm{Ab}$ & $10,75 \mathrm{Bab}$ & $2,05 \mathrm{Bb}$ & $11,98 \mathrm{~b}$ \\
\hline Z. mays + Ipomoea grandifolia & $6,43 \mathrm{Bb}$ & $21,03 \mathrm{Ab}$ & $20,90 \mathrm{Aa}$ & $11,09 \mathrm{ABab}$ & $14,86 \mathrm{~b}$ \\
\hline Z. mays + Hyptis suaveolens & $8,04 \mathrm{Bb}$ & $28,08 \mathrm{Ab}$ & 9,92 Bab & $5,83 \mathrm{Bb}$ & $12,97 \mathrm{~b}$ \\
\hline Z. mays + Conyza canadensis & $7,05 \mathrm{Bb}$ & $16,99 \mathrm{ABb}$ & $7,22 \mathrm{Bb}$ & $19,41 \mathrm{Aa}$ & $12,63 \mathrm{~b}$ \\
\hline Z. mays + Bidens pilosa & $5,58 \mathrm{Bb}$ & $25,30 \mathrm{Ab}$ & $12,72 \mathrm{Bab}$ & $6,93 \mathrm{Bb}$ & $12,63 \mathrm{~b}$ \\
\hline Média & $12,12 \mathrm{~B}$ & $31,88 \mathrm{~A}$ & $12,99 \mathrm{~B}$ & $8,64 \mathrm{~B}$ & \\
\hline CV (\%) & \multicolumn{5}{|c|}{37,68} \\
\hline \multirow{2}{*}{ Espécies } & \multicolumn{4}{|c|}{ S (mg planta $\left.{ }^{-1}\right)$} & \multirow{2}{*}{ Média } \\
\hline & $\mathbf{A A}$ & $\mathbf{C A}$ & SA & SS & \\
\hline Zea mays & $25,13 \mathrm{Ab}$ & $25,69 \mathrm{Aa}$ & $13,98 \mathrm{Ba}$ & $13,54 \mathrm{Ba}$ & $20,13 \mathrm{a}$ \\
\hline Z. mays + Urochloa brizantha & $7,02 \mathrm{Bd}$ & $13,48 \mathrm{Ab}$ & 7,99 Bbc & $6,54 \mathrm{Bb}$ & $8,76 \mathrm{c}$ \\
\hline Z. mays + Ipomoea grandifolia & $16,16 \mathrm{Ac}$ & $11,24 \mathrm{BCb}$ & $13,02 \mathrm{ABa}$ & $8,28 \mathrm{Cb}$ & $12,18 \mathrm{~b}$ \\
\hline Z. mays + Hyptis suaveolens & $45,49 \mathrm{Aa}$ & $11,58 \mathrm{Cb}$ & $6,92 \mathrm{Dbc}$ & $16,51 \mathrm{Ba}$ & $20,13 \mathrm{a}$ \\
\hline Z. mays + Conyza canadensis & $16,28 \mathrm{Ac}$ & $10,55 \mathrm{Bb}$ & $5,50 \mathrm{Cc}$ & $15,66 \mathrm{Aa}$ & $12,00 \mathrm{~b}$ \\
\hline Z. mays + Bidens pilosa & $12,61 \mathrm{Ac}$ & $15,02 \mathrm{Ab}$ & $10,56 \mathrm{Aab}$ & $4,28 \mathrm{Bb}$ & $10,62 \mathrm{bc}$ \\
\hline Média & $20,45 \mathrm{~A}$ & $14,59 \mathrm{~B}$ & $9,66 \mathrm{C}$ & $10,80 \mathrm{C}$ & \\
\hline CV (\%) & & & 19,09 & & \\
\hline
\end{tabular}

${ }^{(1)} \mathrm{AA}$ - com correção de acidez utilizando silicato de cálcio e magnésio e adubação; CA - com correção de acidez utilizando calcário e adubação; SA - sem correção de acidez, mas com adubação; SS - sem correção de acidez e sem adubação. ${ }^{(2)}$ Médias seguidas pela mesma letra maiúscula na linha e minúscula na coluna não diferem entre si pelo teste de Duncan $(p>0,05)$.

Fonte: Elaboração dos autores.

Maior interferência das plantas daninhas, nos manejos de fertilidade do solo estudados, foi causada no solo com adição de silicato de cálcio e magnésio e adubação pelo convívio do milho com U. brizantha e I. grandifolia, com redução média de $59 \%$ no acúmulo de $\mathrm{N}$ em relação à cultura (Tabela 3). Assim como no milho, o $\mathrm{N}$ é o principal macronutriente limitante na produtividade das pastagens, principalmente aquelas formadas por espécies do gênero Urochloa (CECATO et al., 2004). Portanto, conforme constatado por Rajcan e Swanton (2001), a presença de um competidor durante o desenvolvimento e crescimento inicial do milho pode alterar a disponibilidade de $\mathrm{N}$ no solo e sua distribuição na planta. Além disso, espécies que possuem características morfofisiológicas semelhantes geralmente apresentam as mesmas exigências de recursos do meio, tornando a competição mais intensa e as reduções de rendimento mais elevadas (SILVA; DURIGAN,
2006). Em trabalho desenvolvido por Cury et al. (2012), U. brizantha demonstrou ser a espécie com maior capacidade de competição porque afetou negativamente o conteúdo de nutrientes em todos os componentes vegetativos da cultura do milho e ainda apresentou elevados valores desse índice sob competição, o que também foi verificado neste estudo (Tabela 4).

Quanto ao efeito da competição no conteúdo de $\mathrm{P}$ na parte aérea de milho, observaram-se maiores reduções causadas pelas espécies $U$. brizantha nos solos com adição de silicato de cálcio e magnésio e adubação (AA) e sem correção de acidez e sem adubação (SS), da ordem de 75 e $52 \%$, respectivamente e pela espécie $C$. canadensis no solo corrigido com o calcário, com redução média de $74 \%$. No solo sem correção de acidez, mas com adubação somente $I$. grandifolia não mostrou-se competitiva a ponto de interferir no acúmulo de $\mathrm{P}$ pelo milho (Tabela 3 ). 
Nos solos com adição de silicato de cálcio e magnésio e adubação, e calcário e adubação $U$. brizantha e I. grandifolia, juntamente com $H$. suaveolens neste último apresentaram forte concorrência na extração de K levando ao menor acúmulo pelas plantas de milho em comparação à testemunha, enquanto nos solos sem correção de acidez e menos férteis (SA e SS), as plantas que mais competiram foram $B$. pilosa e $H$. suaveolens em SS e C. canadensis juntamente com B. pilosa em SA (Tabela 3). Ronchi et al. (2003) constaram que o conteúdo relativo de macro e micronutrientes na parte aérea de plantas de café foi severamente reduzido devido à interferência de $B$. pilosa, sendo o acúmulo apresentado por esta espécie nestas condições extremamente elevado.

$\mathrm{O}$ conteúdo de $\mathrm{Ca}, \mathrm{Mg}$ e $\mathrm{S}$ na parte aérea das plantas de milho também foi reduzido pela interferência das plantas daninhas, de modo diferenciado nos manejos de fertilidade do solo. Por meio desses resultados verificou-se que, a depender da condição de solo, uma planta daninha se destaca em relação à outra se mostrando mais agressiva e melhor competidora (Tabela 3). Segundo Radosevich, Holt e Ghersa (1996), as plantas são consideradas boas competidoras quando utilizam um recurso rapidamente e são capazes de continuar a crescer mesmo com baixos níveis deste.

Avaliando o desempenho das plantas daninhas em competição com o milho, verificou-se que a habilidade competitiva dessas em extrair e acumular macronutrientes está mais condicionada à adubação do que à adição de fontes de corretivo de acidez, uma vez que, através da média geral, maiores acúmulos foram observados naqueles solos que possuem esta característica em comum (Tabela 4).

Tabela 4. Conteúdo de macronutrientes na parte aérea de Urochloa brizantha, Ipomoea grandifolia, Hyptis suaveolens, Conyza canadensis e Bidens pilosa cultivadas sob interferência de plantas de milho em solo com diferentes manejos de fertilidade.

\begin{tabular}{|c|c|c|c|c|c|}
\hline \multirow{2}{*}{ Espécies } & \multicolumn{4}{|c|}{$\mathbf{N}\left(\right.$ mg planta $\left.^{-1}\right)$} & \multirow{2}{*}{ Média } \\
\hline & $\mathbf{A} \mathbf{A}^{(1)}$ & CA & SA & SS & \\
\hline Urochloa brizantha+Zea mays & $216,73 \mathrm{Ab}^{(2)}$ & $57,28 \mathrm{Bb}$ & $106,65 \mathrm{Bb}$ & $237,92 \mathrm{Aa}$ & $154,65 \mathrm{~b}$ \\
\hline Ipomoea grandifolia $+Z$. mays & $61,13 \mathrm{Ac}$ & $94,17 \mathrm{Ab}$ & $36,65 \mathrm{Abc}$ & $18,22 \mathrm{Ab}$ & $52,54 \mathrm{~cd}$ \\
\hline Hyptis suaveolens $+Z$. mays & $154,89 \mathrm{Ab}$ & $48,88 \mathrm{Bb}$ & $63,65 \mathrm{Bbc}$ & $35,34 \mathrm{Bb}$ & $75,69 \mathrm{c}$ \\
\hline Conyza canadensis $+Z$. mays & $51,20 \mathrm{Ac}$ & $35,69 \mathrm{Ab}$ & $20,09 \mathrm{Ac}$ & $5,53 \mathrm{Ab}$ & $28,13 \mathrm{~d}$ \\
\hline Bidens pilosa + Z. mays & $364,04 \mathrm{Aa}$ & $281,09 \mathrm{BCa}$ & $335,63 \mathrm{ABa}$ & $214,99 \mathrm{Ca}$ & $298,94 \mathrm{a}$ \\
\hline Média & $169,60 \mathrm{~A}$ & $103,42 \mathrm{~B}$ & $112,53 \mathrm{~B}$ & $102,40 \mathrm{~B}$ & \\
\hline CV (\%) & & & 37,90 & & \multirow{3}{*}{ Média } \\
\hline \multirow{2}{*}{ Espécies } & \multicolumn{4}{|c|}{$\mathbf{P}\left(\mathrm{mg} \mathrm{planta}^{-1}\right)$} & \\
\hline & AA & CA & SA & SS & \\
\hline Urochloa brizantha+Zea mays & $16,07 \mathrm{ABb}$ & $8,55 \mathrm{Cbc}$ & $19,13 \mathrm{Ab}$ & $14,02 \mathrm{Ba}$ & $14,44 \mathrm{~b}$ \\
\hline Ipomoea grandifolia $+Z$. mays & $2,81 \mathrm{BCc}$ & $8,90 \mathrm{Ab}$ & 6,68 Abcd & $1,00 \mathrm{Cb}$ & $4,84 \mathrm{~cd}$ \\
\hline Hyptis suaveolens $+Z$. mays & $5,53 \mathrm{Abc}$ & $6,92 \mathrm{ABbc}$ & $9,08 \mathrm{Ac}$ & $3,57 \mathrm{Bb}$ & $6,28 \mathrm{c}$ \\
\hline Conyza canadensis $+Z$. mays & $3,46 \mathrm{Ac}$ & $3,67 \mathrm{Ac}$ & $3,88 \mathrm{Ad}$ & $0,50 \mathrm{Ab}$ & $2,88 \mathrm{~d}$ \\
\hline Bidens pilosa $+Z$. mays & $22,48 \mathrm{Ca}$ & $38,90 \mathrm{Ba}$ & $57,48 \mathrm{Aa}$ & $11,94 \mathrm{Db}$ & $32,71 \mathrm{a}$ \\
\hline Média & $10,07 \mathrm{C}$ & $13,39 \mathrm{Ba}$ & $19,25 \mathrm{~A}$ & $6,21 \mathrm{Da}$ & \\
\hline CV (\%) & & & 23,80 & & \\
\hline
\end{tabular}


continuação

\begin{tabular}{|c|c|c|c|c|c|}
\hline \multirow{2}{*}{ Espécies } & \multicolumn{4}{|c|}{$K\left(\right.$ mg planta $\left.{ }^{-1}\right)$} & \multirow{2}{*}{ Média } \\
\hline & AA & CA & SA & SS & \\
\hline Urochloa brizantha+Zea mays & $152,17 \mathrm{Bb}$ & $77,16 \mathrm{Cb}$ & $175,96 \mathrm{Bb}$ & $255,17 \mathrm{Aa}$ & $165,11 \mathrm{~b}$ \\
\hline Ipomoea grandifolia $+Z$. mays & $36,53 \mathrm{Ac}$ & $77,55 \mathrm{Ab}$ & $60,01 \mathrm{Ac}$ & $18,03 \mathrm{Ac}$ & $48,03 \mathrm{~cd}$ \\
\hline Hyptis suaveolens $+Z$. mays & $84,18 \mathrm{Ac}$ & $70,88 \mathrm{Ab}$ & 77,39 Ac & $60,57 \mathrm{Ac}$ & $73,25 \mathrm{c}$ \\
\hline Conyza canadensis $+Z$. mays & $31,65 \mathrm{Ac}$ & $36,57 \mathrm{Ab}$ & $21,19 \mathrm{Ac}$ & $8,44 \mathrm{Ac}$ & $24,46 \mathrm{~d}$ \\
\hline Bidens pilosa + Z. mays & $256,02 \mathrm{Ba}$ & $215,23 \mathrm{BCa}$ & $320,95 \mathrm{Aa}$ & $176,13 \mathrm{Cb}$ & $242,08 \mathrm{a}$ \\
\hline Média & $112,11 \mathrm{AB}$ & $95,47 \mathrm{~B}$ & $131,10 \mathrm{~A}$ & $103,67 \mathrm{AB}$ & \\
\hline CV (\%) & & & 34,37 & & \multirow{3}{*}{ Média } \\
\hline \multirow{2}{*}{ Espécies } & \multicolumn{4}{|c|}{ Ca (mg planta $\left.{ }^{-1}\right)$} & \\
\hline & $\mathbf{A A}$ & CA & SA & SS & \\
\hline Urochloa brizantha+Zea mays & $144,19 \mathrm{Aa}$ & $70,97 \mathrm{Bb}$ & $132,69 \mathrm{Ab}$ & $53,99 \mathrm{Ba}$ & $100,46 \mathrm{~b}$ \\
\hline Ipomoea grandifolia $+Z$. mays & $7,25 \mathrm{Ac}$ & $23,35 \mathrm{Ac}$ & $15,91 \mathrm{Ad}$ & $2,98 \mathrm{Ac}$ & $11,77 \mathrm{~d}$ \\
\hline Hyptis suaveolens $+Z$. mays & $46,64 \mathrm{Ab}$ & $37,52 \mathrm{Abc}$ & $58,70 \mathrm{Ac}$ & $10,06 \mathrm{Bbc}$ & $38,23 \mathrm{c}$ \\
\hline Conyza canadensis $+Z$. mays & $28,08 \mathrm{Abc}$ & $23,35 \mathrm{Ac}$ & $23,76 \mathrm{Ad}$ & $1,26 \mathrm{Ac}$ & $19,11 \mathrm{~d}$ \\
\hline Bidens pilosa $+Z$. mays & $132,25 \mathrm{Ca}$ & $320,94 \mathrm{Ba}$ & $420,89 \mathrm{Aa}$ & $38,51 \mathrm{Dab}$ & $228,14 \mathrm{a}$ \\
\hline Média & $71,68 \mathrm{C}$ & $94,74 \mathrm{~B}$ & $130,39 \mathrm{~A}$ & $21,36 \mathrm{D}$ & \\
\hline CV (\%) & & & 22,55 & & \\
\hline \multirow{2}{*}{ Espécies } & \multicolumn{4}{|c|}{ Mg $\left(\mathrm{mg} \mathrm{planta}^{-1}\right)$} & \multirow{2}{*}{ Média } \\
\hline & AA & CA & SA & SS & \\
\hline Urochloa brizantha + Zea mays & $41,72 \mathrm{Aa}$ & $13,69 \mathrm{Cb}$ & $27,52 \mathrm{Bb}$ & $20,37 \mathrm{Db}$ & $25,83 \mathrm{~b}$ \\
\hline Ipomoea grandifolia $+Z$. mays & $4,44 \mathrm{BCc}$ & $15,92 \mathrm{Ab}$ & $9,00 \mathrm{Bc}$ & $1,92 \mathrm{Cc}$ & $7,82 \mathrm{c}$ \\
\hline Hyptis suaveolens $+Z$. mays & $7,37 \mathrm{Ac}$ & $10,78 \mathrm{Abc}$ & $7,73 \mathrm{Ac}$ & $6,16 \mathrm{Ac}$ & $8,01 \mathrm{c}$ \\
\hline Conyza canadensis $+Z$. mays & $4,91 \mathrm{Ac}$ & $6,27 \mathrm{Ac}$ & $4,10 \mathrm{Ac}$ & $0,81 \mathrm{Ac}$ & $4,02 \mathrm{~d}$ \\
\hline Bidens pilosa $+Z$. mays & $22,88 \mathrm{Bb}$ & $50,69 \mathrm{Aa}$ & $50,25 \mathrm{Aa}$ & $27,30 \mathrm{Ba}$ & $37,78 \mathrm{a}$ \\
\hline Média & $16,26 \mathrm{~B}$ & $19,47 \mathrm{~A}$ & $19,72 \mathrm{~A}$ & $11,31 \mathrm{C}$ & \\
\hline CV (\%) & & & 20,35 & & \\
\hline \multirow{2}{*}{ Espécies } & \multicolumn{4}{|c|}{ S $\left(\right.$ mg planta $\left.^{-1}\right)$} & \multirow{2}{*}{ Média } \\
\hline & $\mathbf{A A}$ & CA & SA & SS & \\
\hline Urochloa brizantha + Zea mays & $15,53 \mathrm{Bb}$ & $11,96 \mathrm{Bb}$ & $12,16 \mathrm{Bc}$ & $23,68 \mathrm{Aa}$ & $15,83 \mathrm{~b}$ \\
\hline Ipomoea grandifolia $+Z$. mays & $3,22 \mathrm{Bd}$ & $8,55 \mathrm{Abc}$ & $4,80 \mathrm{ABd}$ & $1,34 \mathrm{Bb}$ & $4,47 \mathrm{~d}$ \\
\hline Hyptis suaveolens $+Z$. mays & $10,47 \mathrm{Bc}$ & $8,07 \mathrm{BCbc}$ & $29,97 \mathrm{Ab}$ & $5,00 \mathrm{Cb}$ & $13,38 \mathrm{c}$ \\
\hline Conyza canadensis $+Z$. mays & $3,56 \mathrm{Ad}$ & $4,02 \mathrm{Ac}$ & $4,27 \mathrm{Ad}$ & $0,74 \mathrm{Ab}$ & $3,15 \mathrm{~d}$ \\
\hline Bidens pilosa $+Z$. mays & $20,64 \mathrm{Ca}$ & $78,53 \mathrm{Aa}$ & $44,59 \mathrm{Ba}$ & $23,50 \mathrm{Ca}$ & $41,82 \mathrm{a}$ \\
\hline Média & $10,68 \mathrm{C}$ & $22,22 \mathrm{~A}$ & $19,16 \mathrm{~B}$ & $10,85 \mathrm{C}$ & \\
\hline CV (\%) & & & 18,37 & & \\
\hline
\end{tabular}

(1) AA - com correção de acidez utilizando silicato de cálcio e magnésio e adubação; CA - com correção de acidez utilizando calcário e adubação; SA - sem correção de acidez, mas com adubação; SS - sem correção de acidez e sem adubação. ${ }^{(2)}$ Médias seguidas pela mesma letra maiúscula na linha e minúscula na coluna não diferem entre si pelo teste de Duncan $(\mathrm{p}>0,05)$.

Fonte: Elaboração dos autores.

U. brizantha em competição com o milho no solo sem correção de acidez e sem adubação (SS) chegou a acumular cerca de 4, 2, 3 e 2 vezes mais N, P, K e $\mathrm{S}$, respectivamente, do que nos solos com adição de calcário (Tabela 4), mostrando sua capacidade de extração de nutrientes frente a situações de recursos limitados. Esta espécie se destacou em relação às demais plantas daninhas com maior conteúdo de $\mathrm{K}$ e P na parte aérea na condição de solo SS (Tabela 4).

Dentre as plantas daninhas em competição, apesar do potencial competitivo apresentado por $U$. brizantha em convivência com o milho (Tabela 2), B. pilosa foi a espécie que extraiu e acumulou 
em média e na maioria das condições de solo maior quantidade de macronutrientes (Tabela 4). De acordo com Santos e Cury (2011), Bidens pilosa pode promover elevada extração de nutrientes e quanto maior a extração, maior será o potencial competitivo com a cultura de interesse, sendo capaz de acumular teores e quantidades totais relativamente altos de nitrogênio, fósforo e micronutrientes. A capacidade competitiva de uma espécie pode ser determinada pelo volume de solo explorado pelo sistema de raízes, a eficiência no uso da água e a capacidade de extração de recursos do solo (PROCÓPIO et al., 2004), podendo também estar diretamente relacionada à capacidade associativa ou habilidade das plantas daninhas em alterar a microbiota do solo em prol de seu favorecimento (MELO, 2012).

O milho sofreu maior concorrência com as plantas daninhas na extração de nutrientes quando cultivado no solo sem correção de acidez e adubação, apresentando maior capacidade competitiva no solo corrigido com calcário. Silicato de cálcio e magnésio e calcário exerceram influência variável sobre o conteúdo de macronutrientes nas folhas das plantas daninhas em monocultivo ou sob interferência. Urochloa brizantha foi a espécie de planta daninha que causou maior redução no conteúdo de nutrientes na parte aérea das plantas de milho, independente do manejo de fertilidade do solo. Plantas daninhas apresentaram, a depender do manejo de fertilidade de solo, potenciais diferenciados para ciclagem dos macronutrientes, com destaque para Bidens pilosa que acumulou elevado teor de nutrientes em convivência com o milho e livre de interferência.

\section{Agradecimentos}

A Coordenação de Aperfeiçoamento de Pessoal de Nível Superior (CAPES), ao Conselho Nacional de Desenvolvimento Científico e Tecnológico (CNPq) e a Fundação de Amparo à Pesquisa do Estado de Minas Gerais (FAPEMIG), pela concessão da bolsa de mestrado e apoio financeiro para execução do trabalho.

\section{Referências}

ALVES, V. M. C.; VASCONCELLOS, C. A.; FREIRE, F. M.; PITTA, G. V. E.; FRANÇA, G. E.; RODRIGUES FILHO, A.; ARAÚJO, J. M.; VIEIRA, J. R.; LOUREIRO, J. E. Sugestões de adubação para diferentes culturas em Minas Gerais - Milho. In: RIBEIRO, A. C.; GUIMARÃES, P. T. G.; VENEGAS, V. H. A. (Ed.). Recomendações para o uso de corretivos e fertilizantes em Minas Gerais: $5^{\mathrm{a}}$ aproximação. Viçosa: CFSEMG, 1999. cap. 18, p. 313-316.

BRAGA, J. M.; DEFELIPO, B. V. Determinação espectrofotométrica de $\mathrm{P}$ em extratos de solo e material vegetal. Revista Ceres, Viçosa, v. 21, n. 1, p. 73-85, 1974.

CARVALHO, L. B.; BIANCO, S.; PITELLI, R. A.; BIANCO, M. S. Estudo comparativo do acúmulo de massa seca e macronutrientes por plantas de milho Var. BR-106 e Brachiaria plantaginea. Planta Daninha, Viçosa, v. 25, n. 2, p. 293-301, 2007.

CECATO, U.; PEREIRA, L. A. F.; GALBEIRO, S.; SANTOS, G. T.; DAMASCENO, J. C.; MACHADO, A. O. Influência da adubação nitrogenada e fosfatada na produção, na rebrota e no perfilhamento do capimmarandú (Brachiaria brizantha [Hochst] stapf. Cv. Marandú). Acta Scientiarum. Animal Science, Maringá, v. 26, n. 3, p. 399-407, 2004.

CONSTANTIN, J.; OLIVEIRA JÚNIOR, R. S.; CAVALIERI, S. D.; ARANTES, J. G. Z.; ALONSO, D. G.; ROSO, A. C.; COSTA, J. M. Interação entre sistemas de manejo e de controle de plantas daninhas em pós-emergência afetando o desenvolvimento e a produtividade do milho. Planta Daninha, Viçosa, v. 25, n. 3, p. 513-520, 2007.

CURY, J. P.; SANTOS, J. B.; SILVA, E. B.; BYRRO, E. C. M.; BRAGA, R. R.; CARVALHO, F. P.; VALADÃO SILVA, D. Acúmulo e partição de nutrientes de cultivares de milho em competição com plantas daninhas. Planta Daninha, Viçosa, v. 30, n. 2, p. 287-296, 2012.

KARAM, D.; MELHORANÇA, A. L.; OLIVEIRA, M. F. Plantas daninhas na cultura do milho. Sete Lagoas: EMBRAPA-CNPMS, 2006. 7 p.

MALAVOLTA, E.; VITTI, G. C.; OLIVEIRA, A. S. Avaliação do estado nutricional das plantas: princípios e aplicações. 2. ed. São Paulo: Potafos, 1997. 319 p.

MELO, C. A. D. Atividade microbiana e interferência de plantas daninhas na cultura do milho em solo com diferentes manejos de fertilidade. 2012. Dissertação (Mestrado em Fitotecnia) - Programa de Pós-Graduação em Fitotecnia Universidade Federal de Viçosa, Viçosa. 
PROCÓPIO, S. O.; SANTOS, J. B.; SILVA, A. A.; DONAGEMMA, G. K.; MENDONÇA, E. S. Ponto de murcha permanente de soja, feijão e plantas daninhas. Planta Daninha, Viçosa, v. 22, n. 1, p. 35-41, 2004.

RADOSEVICH, S. R.; HOLT, J. S.; GHERSA, C. Physiological aspects of competition. In: RADOSEVICH, S. R.; HOLT, J. S.; GHERSA, C. (Ed.). Weed ecology: implicatios for manegements. 2. ed. New York: John Willey and Sons, 1996. cap. 6, p. 217-301.

RAJCAN, I.; SWANTON, C. J. Understanding maizeweed competition: resource competition, light quality and the whole plant. Field Crops Research, Amsterdam, v. 71, n. 2, p. 139-150, 2001.

RAMOS, L. A.; NOLLA, A.; KORNDÖRFER, G. H.; PEREIRA, H. S.; CAMARGO, M. S. Reatividade de corretivos da acidez e condicionadores de solo em colunas de lixiviação. Revista Brasileira de Ciência do Solo, Viçosa, v. 30, n. 5, p. 849-857, 2006.

RONCHI, C. P.; TERRA, A. A.; SILVA, A. A.; FERREIRA, L. R. Acúmulo de nutrientes pelo cafeeiro sob interferência de plantas daninhas. Planta Daninha, Viçosa, v. 21, n. 2, p. 219-227, 2003.
SANTOS, J. B.; CURY, J. P. Picão-preto: uma planta daninha especial em solos tropicais. Planta Daninha, Viçosa, v. 29, p. 1159-1171, 2011. Número Especial.

SARRUGE, J. R.; HAAG, H. P. Análises químicas em plantas. Piracicaba: ESALQ, Departamento de Química, 1974, $56 \mathrm{p}$.

SILVA, A. A.; FERREIRA, F. A.; FERREIRA, L. R.; SANTOS, J. B. Biologia de plantas daninhas. In: SILVA, A. A.; SILVA, J. F. (Ed.). Tópicos em manejo de plantas daninhas. Viçosa: Editora UFV, 2007. cap. 1, p. 17-61.

SILVA, M. R. M.; DURIGAN, J. C. Períodos de interferência das plantas daninhas na cultura do arroz de terras altas. I - Cultivar IAC 202. Planta Daninha, Viçosa, v. 24, n. 4, p. 685-694, 2006.

YASUHARA, T.; NOKIHARA, K. High-throughput analysis of total nitrogen content that replaces the classic Kjeldahl method. Journal of Agricultural and Food Chemistry, Washington, v. 49, n. 10, p. 4581-4583, 2001.

ZANINE, A. M.; SANTOS, E. D. Competição entre espécies de plantas - uma revisão. Revista da FZVA, Uruguaiana, v. 11, n. 1, p. 103-122, 2004. 
